\title{
AEROVIGILA_OMA - Aplicación informática de vigilancia para oficinas meteorológicas de aeródromo
}

https://doi.org/10.31978/639-19-010-0.089

\author{
Javier Mediavilla González¹ (jmediavillag@aemet.es) \\ José Manuel López Pérez ${ }^{1}$ (jolopezp@aemet.es)
}

${ }^{1}$ AEMET / Delegación Territorial en la Comunidad Valenciana

\begin{abstract}
RESUMEN
Entre las funciones de las oficinas meteorológicas de aeródromo se encuentra la vigilancia meteorológica continua sobre el aeródromo. El objetivo de esta aplicación es ayudar al observador en esta función. Presenta de forma automática y actualiza periódicamente los datos e informes meteorológicos y activa una alerta acústica ante determinados eventos que se pueden configurar. La aplicación está desarrollada con AUTOIT en el entorno operativo Windows.
\end{abstract}

PALABRAS CLAVE: OMA; TAF; METAR; avisos de aeródromo; descargas eléctricas; SIGMET.

\section{INTRODUCCIÓN}

La predicción meteorológica a corto plazo ha evolucionado mucho durante las últimas décadas, llegando a ser muy fiable en el plazo de unos pocos días, gracias en gran parte a las mejoras en los modelos meteorológicos de predicción del tiempo. Estos modelos resuelven un problema de condiciones iniciales en el que, fundamentalmente, se predice el estado de la atmósfera y de la superficie terrestre y se consideran el resto de elementos del sistema climático constantes en el tiempo (singularmente la temperatura del agua del mar). Sin embargo, para predicciones a alcances medio y superiores se requiere al menos el acoplo en los modelos del componente oceánico.

La aplicación presenta de forma automática y actualiza con una periodicidad que se puede elegir, la siguiente información:

- últimos mensajes aeronáuticos TAF y METAR;

- avisos de aeródromo en vigor o próximos a entrar en vigor;

- avisos de fenómenos adversos de meteoalerta en la provincia donde se encuentra el aeropuerto;

- detección de descargas eléctricas en el entorno del aeropuerto en un radio a elección;

- avisos de tormentas observados en las cercanías (WWSP62);

- avisos de rayos observados en las cercanías (WWSP61);

- mensajes aeronáuticos de área SIGMET y AIRMET. 
La aplicación emite una alerta acústica que se puede configurar para:

- emisión de un nuevo aviso de aeródromo;

- TAF NIL;

- METAR NIL;

- nuevo aviso de fenómeno adverso de meteoalerta;

- detección de descargas eléctricas o tormentas en las cercanías;

- notificación a determinados minutos a elección (modo despertador);

- superación de determinados umbrales configurables en el pronóstico TAF;

- nuevo boletín SIGMET y/o AIRMET.

\section{CONFIGURACIÓN INICIAL}

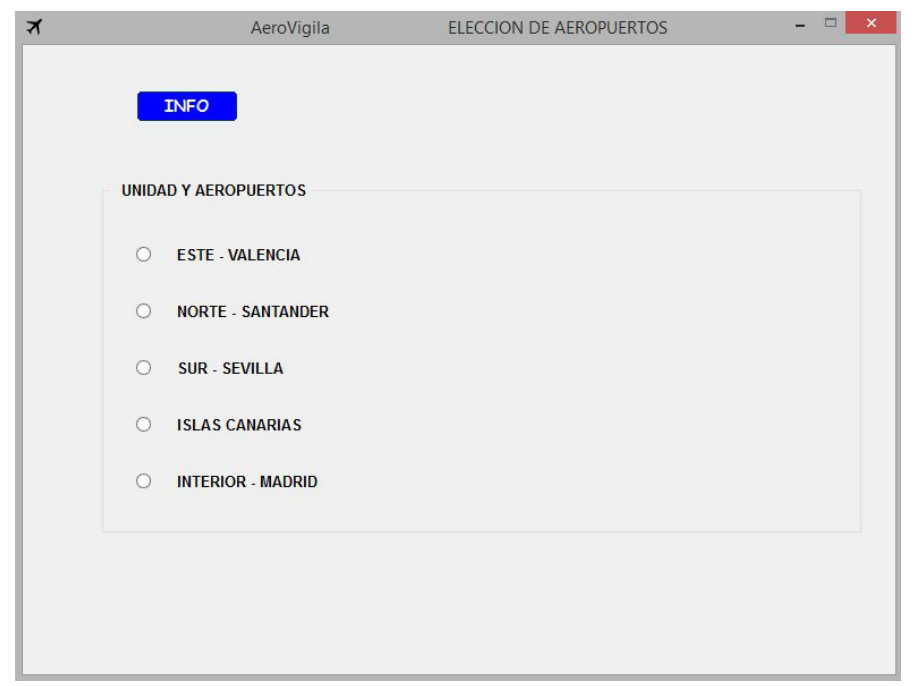

Figura 1. Pantalla de inicio.

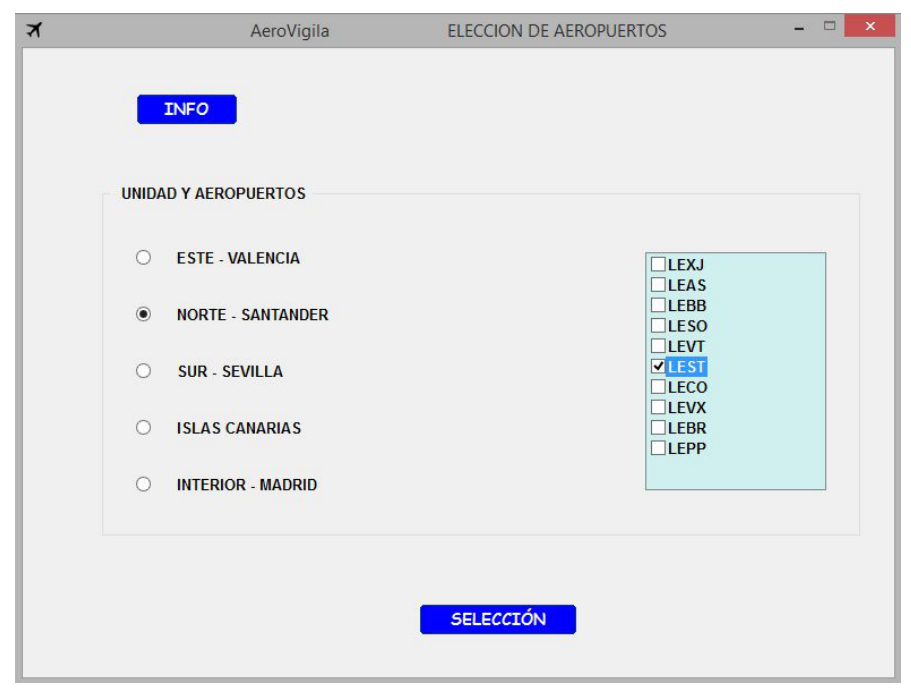

Figura 2. Elección de aeropuertos.
Se elige en la pantalla de inicio (figura 1) el Grupo de Predicción y Vigilancia al que pertenece el aeropuerto.
Aparecen todos los aeropuertos del GPV (figura 2). Se selecciona el aeropuerto o los aeropuertos para realizar la vigilancia. Permite elegir los que se quiera siempre que correspondan al mismo GPV.

Se pulsa el botón SELECCIÓN. La aplicación accede automática y periódicamente a los últimos datos. Presenta en pantalla la información y si es procedente activa la alerta acústica.

El último METAR y el TAF se presentan en pantalla. También si hubiese avisos de aeródromo y de meteoalerta, como puede observarse en la figura 3 . 


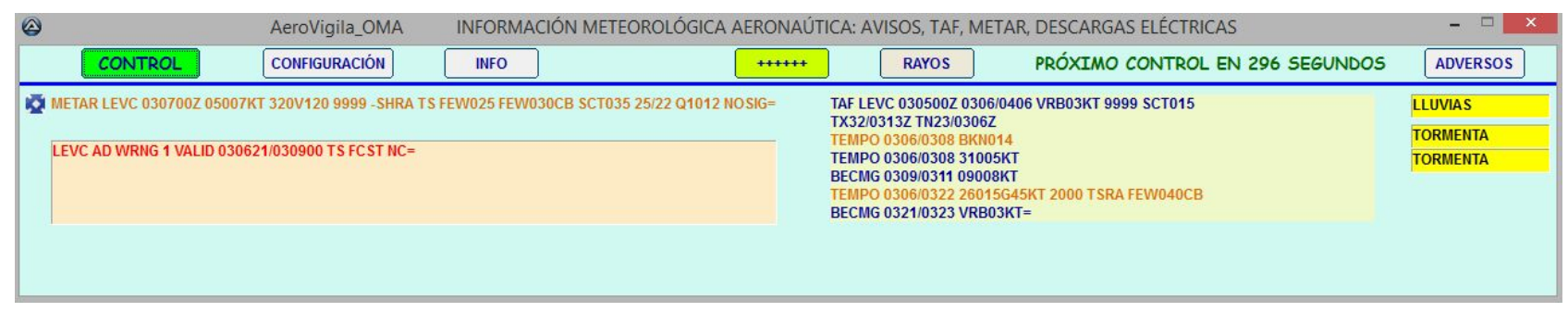

Figura 3. Información del aeropuerto de Valencia, LEVC.

\section{DESCRIPCIÓN DE LAS CARACTERÍSTICAS DE LA APLICACIÓN}

En la parte superior de la ventana de la aplicación están situados los siguientes botones:

\subsection{Botón de control}

Permite actualizar datos sin esperar a que se cumpla el tiempo asignado a la vigilancia periódica.

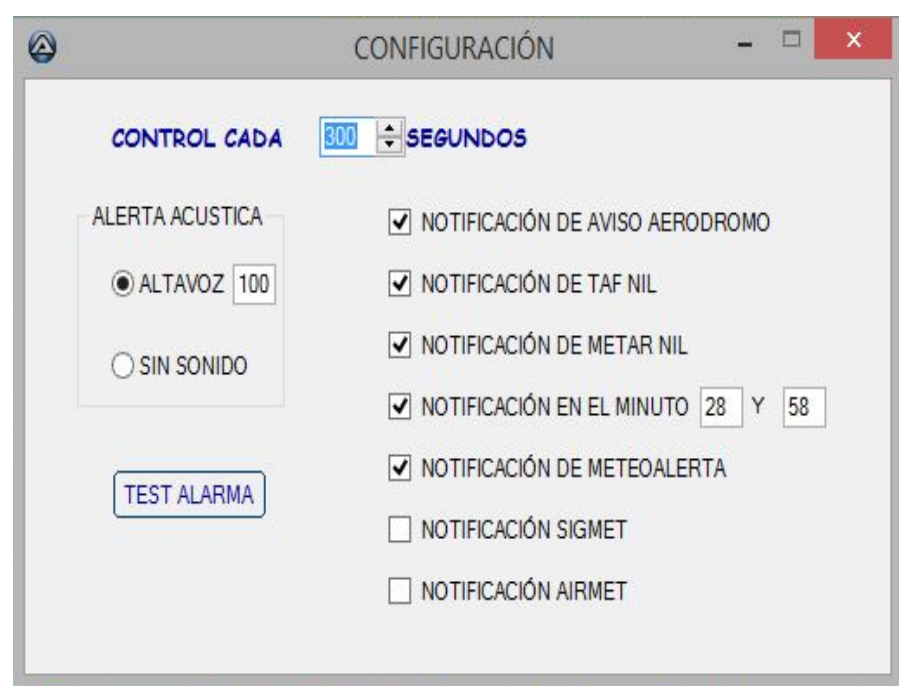

Figura 4. Ventana de configuración de alertas.

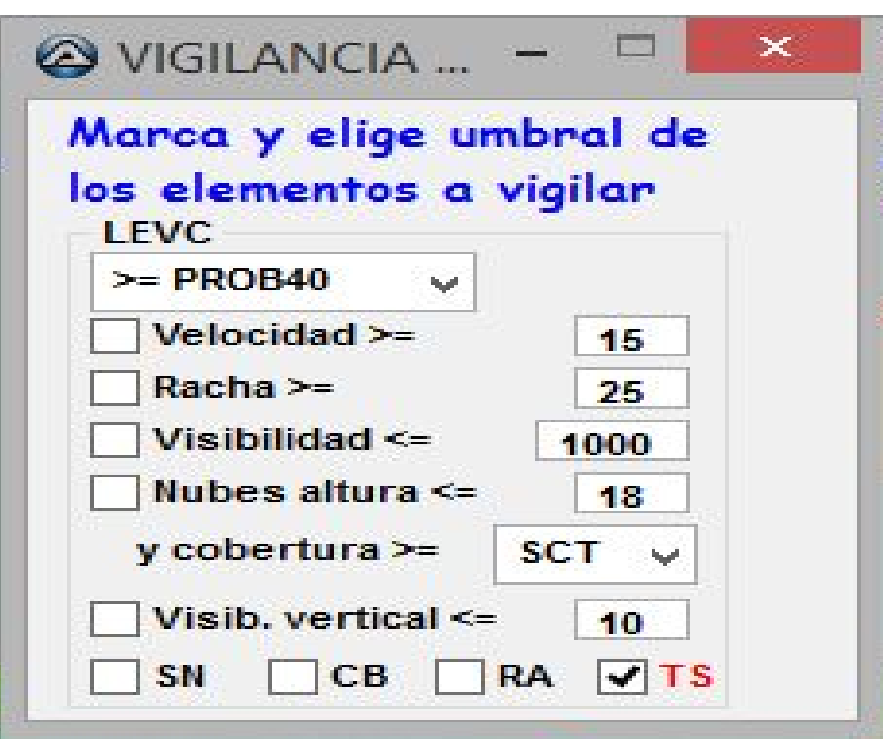

Figura 5. Ventana configuración vigilancia TAF.

\subsection{Botón de configuración}

Abre una ventana (figura 4) para poder elegir los diferentes avisos disponibles y la periodicidad de la vigilancia.

Al iniciar están marcados todos menos notificación de SIGMET y AIRMET. Se pueden marcar o desmarcar según las necesidades de vigilancia. En la notificación en un determinado minuto permite elegir el momento, en el inicio se configura para el minuto 28 y 58 para hacerlo coincidir con la emisión del METAR. Esta ventana no necesita estar abierta para realizar la vigilancia, una vez realizada la selección, se puede cerrar. Sucede lo mismo con las ventanas RAYOS y +++++ .

\subsection{Info}

El botón de información presenta un resumen informativo de la aplicación.

\subsection{Botón ++++++}

Abre una ventana (figura 5) con la que se pueden configurar umbrales para la vigilancia automática del pronóstico TAF y si son superados generará un aviso acústico.

Los elementos a vigilar deben ser marcados. En este caso se han marcado las tormentas, si en el TAF se pronosticaran con una probabilidad mayor del $40 \%$ generará aviso acústico y visual. Los umbrales que aparecen en la ventana se pueden modificar (por ejemplo, en la racha, en el 
inicio es 25 pero se puede modificar a otro valor). Se puede elegir el grado de probabilidad (en el inicio es $\geq$ PROB40). Si hay algún elemento a vigilar el botón ++++++ aparecerá en color verde en vez de gris y si se alcanza el umbral elegido se pondrá rojo. Los aeropuertos de Valencia LEVC, Ibiza LEIB y Zaragoza LEZG inician la aplicación con pronóstico de niebla o PROB40 de niebla. Zaragoza también incluye nubosidad baja BKN o OVC.

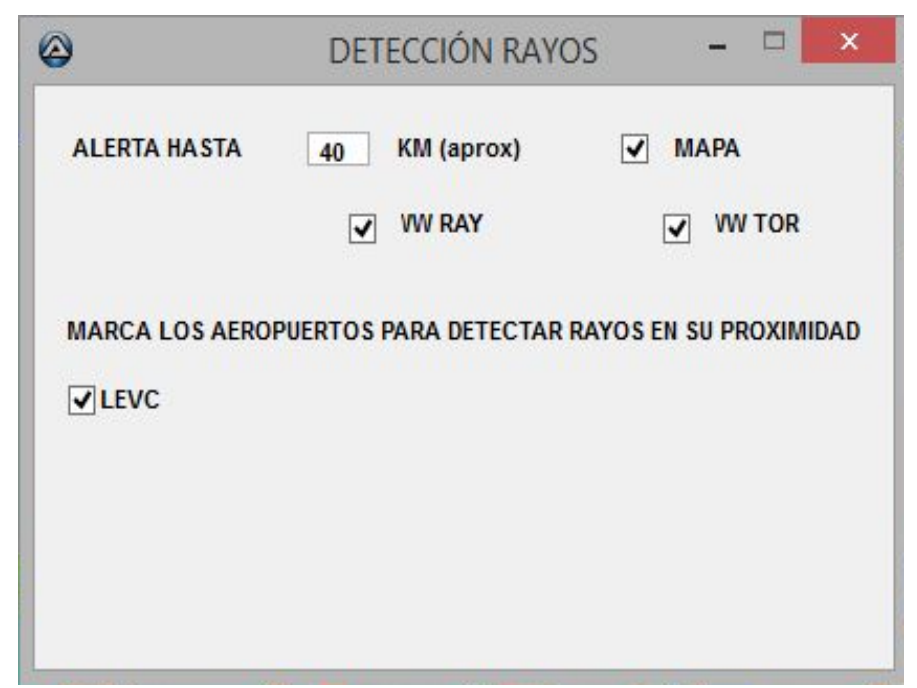

Figura 6. Ventana de configuración de rayos.

\section{5. Botón rayos}

Permite configurar la vigilancia de descargas eléctricas en el entorno del aeropuerto y los boletines de avisos de rayos y tormentas (WWSP61 y WWSP62).

Para realizar la vigilancia de rayos y tormentas hay 3 formas no excluyentes.

\section{5.a. Descargas eléctricas}

Si se quiere realizar la vigilancia de descargas eléctricas en un entorno del aeropuerto, hay que marcar MAPA (figura 6) y como valor de inicio aparece $40 \mathrm{~km}$, que puede ser modificado, y es

la distancia aproximada a la que la aplicación avisa si hay descargas dentro del círculo de ese radio centrado en el aeropuerto en la última hora.

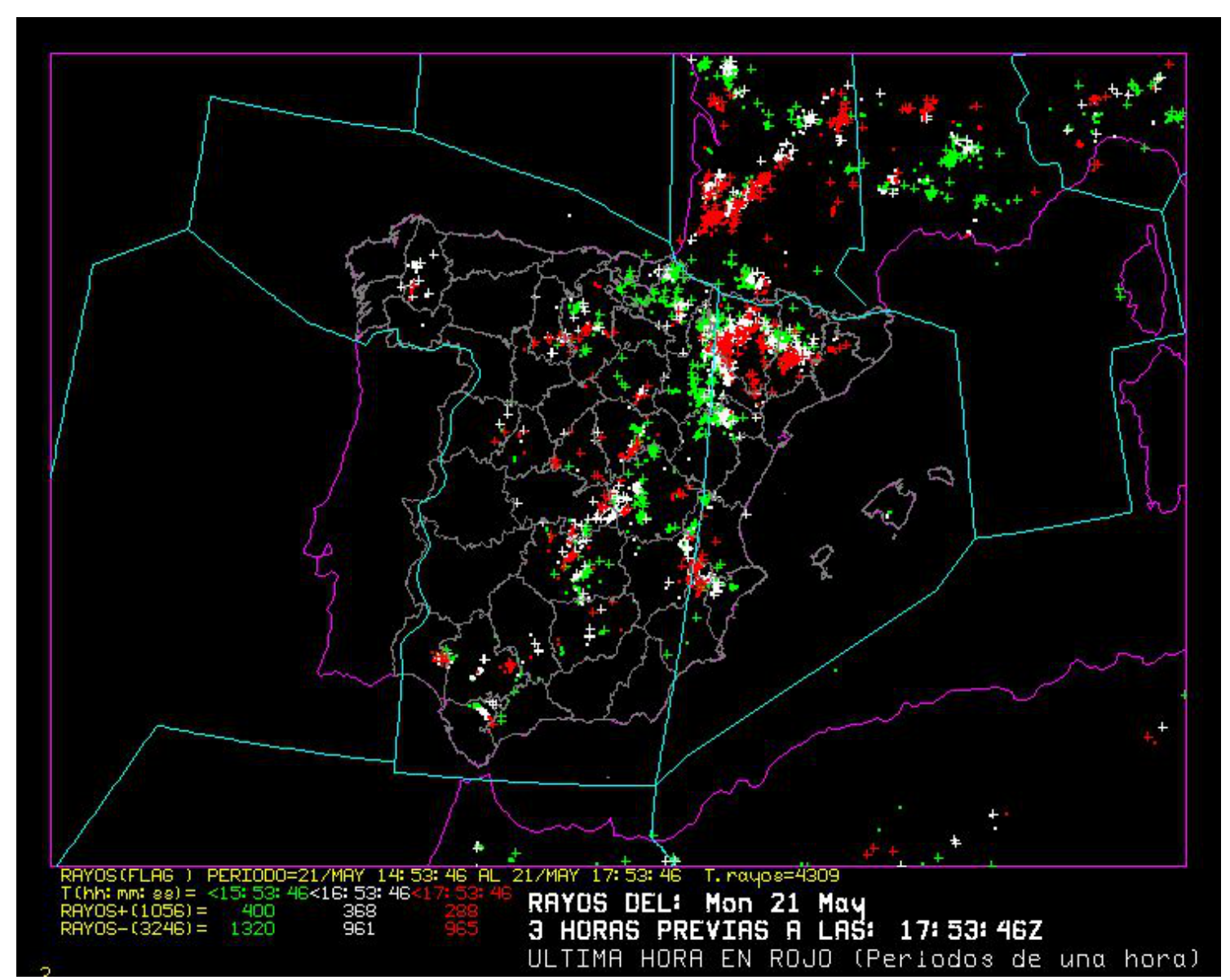

Figura 7. Descargas eléctricas últimas 3 horas. 


\section{5.b. WW RAY}

Si se marca WW RAY se accederá a los boletines de avisos de rayos WWSP61, para los aeropuertos elegidos. Estos boletines están basados en el análisis de datos procedentes de descargas eléctricas. Las especificaciones de usuario de estos avisos se pueden consultar en: http://www0.aemet.es/wwy/paginaweb/ Especificaciones/MPO-ESP-0100.pdf.

\section{5.c. WW TOR}

Marcando WW TOR son los avisos WWSP62 correspondientes a tormentas. Estos boletines están basados en el análisis de datos procedentes de descargas eléctricas y radar. Las especificaciones de usuario de estos avisos se pueden consultar en: http://www0.aemet.es/wwy/paginaweb/Especificaciones/MPO-ESP-0304.pdf.

Pueden marcarse los tres tipos simultáneamente, y una vez cerrada esta ventana, cuando realice la siguiente vigilancia o pulsando el botón CONTROL accederá a los boletines de avisos y al mapa de rayos si han sido elegidos. El aeropuerto, o los aeropuertos si se ha iniciado la aplicación con más de uno, debe estar marcado, permitiendo que se desmarquen o vuelvan a marcar según la necesidad de vigilancia de dicho aeropuerto. El botón rayos inicialmente en color gris, se presentará en verde si se ha marcado algún tipo de vigilancia de rayos. Una vez que realiza la vigilancia si hay algún boletín de aviso o en el mapa se han localizado descargas próximas, el botón rayos se pondrá en color rojo, se activará la alerta acústica y la notificación del tipo de aviso que se ha activado (figura 8).

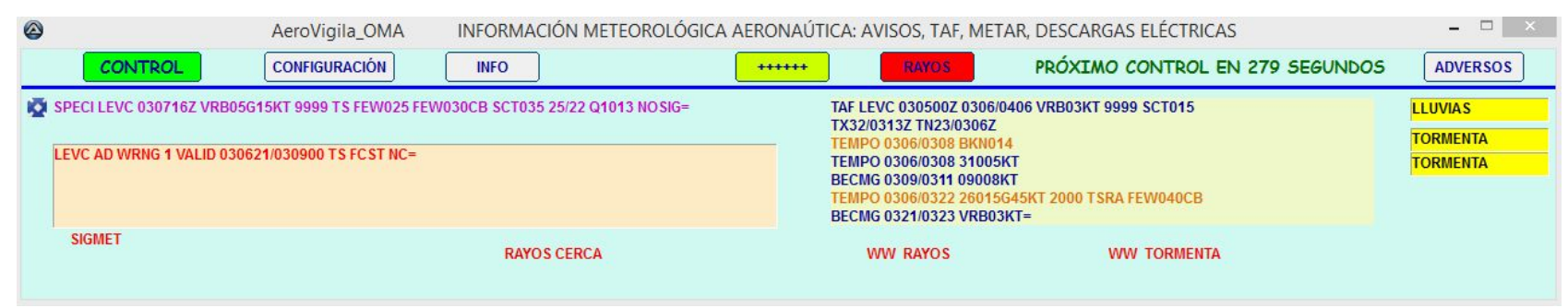

Figura 8. Aplicación con vigilancia de rayos activada.

\subsection{Botón adversos}

Accede a la página web de AEMET de avisos de METEOALERTA para comprobar los avisos que aparecen a la derecha de la ventana de la aplicación y debajo del botón ADVERSOS (figura 8) aparecen los avisos para la provincia del aeropuerto y el color correspondiente.

\subsection{Botón azul}

A la izquierda del METAR aparece un pequeño botón azul, pulsando se accede a la información de las últimas 36 horas de dicho aeropuerto.

\section{ENLACE DE DESCARGA DE LA APLICACIÓN}

La aplicación se puede descargar en el siguiente enlace:

https://www.dropbox.com/s/zfh3ktekuop511t/AeroVigila_OMA_12.exe?dl=0

Para poder utilizar la información es necesario tener acceso a la intranet de AEMET. 


\section{CONCLUSIONES}

El objetivo de la aplicación es automatizar de forma periódica el acceso a la información meteorológica de interés en el trabajo de vigilancia de la OMA, garantizar un rápido conocimiento de los datos relevantes y generar la alerta si son superados los umbrales elegidos. La automatización de determinadas condiciones del aeródromo permite liberar el trabajo de vigilancia de las tareas más repetitivas y reducir el tiempo en que tardan en conocerse los datos relevantes. La capacidad de definir en la aplicación qué información es la relevante, permite generar las alertas acústicas que el observador de la OMA necesita para desarrollar su trabajo.

\section{AGRADECIMIENTOS}

La Oficina Meteorológica de Aeródromo de Manises, Valencia ha realizado las pruebas y sugerido mejoras de la aplicación.

\section{REFERENCIAS}

Conde, M. V., 2008. Especificaciones de usuario de los avisos de tormentas previstas en los aeródromos.

Conde, M. V., 2008. Especificaciones de usuario de los avisos de rayos detectados en los aeródromos.

MEDIAVILLA, J., 2018. AEROVIGILA_OMA —-Aplicación informática de vigilancia para oficinas meteorológicas de aeródromo. Documentación de la aplicación GPV de Valencia.

Portillo, A., 2013. Claves aeronáuticas FM 15 METAR, FM 16 SPECI y FM TAF. 